\title{
LIOUVILLIAN AND ANALYTIC FIRST INTEGRALS FOR THE BRUSSELATOR SYSTEM
}

\author{
JAUME LLIBRE ${ }^{1}$ AND CLÀUDIA VALLS ${ }^{2}$
}

\begin{abstract}
We characterize the Liouvillian and analytic first integrals for the polynomial differential systems of the form $x^{\prime}=a-(b+1) x+x^{2} y, y^{\prime}=b x-x^{2} y$, with $a, b \in \mathbb{R}$, called the Brusselator differential systems.
\end{abstract}

\section{IntRoduCtion AND STATEMENT OF THE MAIN RESUltS}

One of the more classical problems in the qualitative theory of planar differential systems depending on parameters is to characterize the existence or not of first integrals.

We consider the system

$$
x^{\prime}=a-(b+1) x+x^{2} y, \quad y^{\prime}=b x-x^{2} y
$$

that we call the Brusselator, where $x$ and $y$ are complex variables and the prime denotes derivative with respect to the time $t$, which can be either real or complex. Such differential systems appear in several branches of the sciences, mainly in chemistry since it studies a certain chemical reaction (see $[7,9,16]$ for details).

Let $U \subset \mathbb{C}^{2}$ be an open and dense set in $\mathbb{C}^{2}$. We say that the non-constant function $H: \mathbb{C}^{2} \rightarrow \mathbb{C}$ is a first integral of the polynomial vector field $\chi$ on $U$, if $H(x(t), y(t))=$ constant for all values of $t$ for which the solution $(x(t), y(t))$ of $c h i$ is defined on $U$. Clearly $H$ is a first integral of $\chi$ on $U$ if and only if $\chi H=0$ on $U$.

An analytic first integral is a first integral which is an analytic function. As a particular case, a first integral which is a polynomial is called a polynomial first integral.

A Liouvillian first integral is a first integral $H$ which is a Liouvillian function, that is, roughly speaking which can be obtained "by quadratures" of elementary functions. For a precise definition see [20]. The study of the Liouvillian first integrals is a classical problem of the integrability theory of the differential equations which goes back to Liouville, see for details again [20].

As far as we know the Liouvillian and analytic first integrals of some multi-parameter family of planar polynomial differential systems has only been classified for few differential systems, see for instance $[3,8,9,10,11,12,13,14,15,16,17,18]$.

We first study system (1) with $a=0$. In this case changing the independent variable $t$ as follows $x d t=d \tau$, it becomes

$$
\dot{x}=-b-1+x y, \quad \dot{y}=b-x y,
$$

where the dot denotes derivative with respect to $\tau$.

Let $F$ be the Kummer confluent hypergeometric function, and $H_{b}$ be the Hermite function, when $b$ is a positive integer provides the Hermite polynomial of degree $n$. For more details on these two functions see [1].

2010 Mathematics Subject Classification. 34C35, 34D30.

Key words and phrases. Darboux polynomial, exponential factor, Liouvillian first integral, analytic first integral, Brusselator differential systems. 
Proposition 1. System (2) has the analytic first integral

$$
\frac{(b+1)(y+x) F\left(-\frac{b}{2} ; \frac{3}{2} ; \frac{(y+x)^{2}}{2}\right)-x F\left(-\frac{b}{2} ; \frac{1}{2} ; \frac{(y+x)^{2}}{2}\right)}{\sqrt{2} b H_{b-1}\left(\frac{y+x}{\sqrt{2}}\right)-y H_{b}\left(\frac{y+x}{\sqrt{2}}\right)}
$$

It is easy to check that the function given in (3) is an analytic first integral of system (1) with $a=0$ defined in an open and dense set of $\mathbb{C}^{2}$.

Theorem 2. The Brusselator system (1) with $a \neq 0$ has no analytic first integrals.

Theorem 2 is proved in Section 2 .

It follows from Theorem 2 that system (1) with $a \neq 0$ has no Liouvillian first integrals (otherwise it would have analytic first integrals since the last class include in particular the Liouvillian first integrals). However when $a=0$, it could be that for some values of $b$, the first integral given in (3) could be of Liouvillian type and that system (2) could have a Liouvillian first integral. We will see that this can not happen. So, from now on we restrict to system $(2)$.

Let $h(x, y) \in \mathbb{C}[x, y] \backslash \mathbb{C}$. As usual $\mathbb{C}[x, y]$ denotes the ring of all complex polynomials in the variables $x$ and $y$. We say that $h=0$ is an invariant algebraic curve of the vector field $X$ associated to system (2) if it satisfies

$$
(-b-1+x y) \frac{\partial h}{\partial x}+(b-x y) \frac{\partial h}{\partial y}=K h,
$$

the polynomial $K=K(x, y) \in \mathbb{C}[x, y]$ is called the cofactor of $h=0$ and has degree at most 1. We also say that $h$ is a Darboux polynomial of system (1). Note that a polynomial first integral is a Darboux polynomial with zero cofactor.

The invariant algebraic curves are important because a sufficient number of them forces the existence of a first integral. This result is the basis of the Darboux theory of integrability, see for instance $[3,5,8]$. In the next result we characterize all the algebraic invariant curves for system (2).

Proposition 3. The following statements hold for system (2).

(a) It has no Darboux polynomials with zero cofactor.

(b) It has an irreducible Darboux polynomial $h=h(x, y)$ with non-zero cofactor if and only if $b=0$, and in this case $h=y$ and the cofactor is $-x$.

Proposition 3 is proved in Section 3.

An exponential factor $E$ of system (1) is a function of the form $E=\exp (g / h) \notin \mathbb{C}$ with $g, h \in \mathbb{C}[x, y]$ coprime satisfying that

$$
(-b-1+x y) \frac{\partial E}{\partial x}+(b-x y) \frac{\partial E}{\partial y}=L E,
$$

for some polynomial $L=L(x, y)$ of degree at most 1, called the cofactor of $E$.

The existence of exponential factors $\exp (g / h)$ is due to the fact that the multiplicity of the invariant algebraic curve $h=0$ is larger than 1 , and when $h$ is constant, the existence of the exponential factor is due to the multiplicity of the straight line at infinity in the projective plane, for more details see [5].

Proposition 4. System (2) has two exponential factors modulo constants: $e^{x+y}$ with cofactor -1 and $e^{(x+y)^{2}}$ with cofactor $-2(x+y)$.

Proposition 4 is proved in Section 4. 
Theorem 5. System (2) has a Liouvillian first integral if and only if $b=0$ and in this case the Liouvillian first integral is

$$
\frac{1}{y}\left(\sqrt{2 \pi} y \operatorname{Erfi}\left(\frac{-x-y}{\sqrt{2}}\right)+2 e^{\frac{1}{2}(x+y)^{2}}\right)
$$

where Erfi( $z)$ is the imaginary error function, for more details see [1].

Theorem 5 is proved in Section 5.

As a corollary of Theorems 2 and 5 we have the following result.

Corollary 6. System (1) has a analytic first integral if and only if $a=0$. Moreover such first integral is Liouvillian if and only if $b=0$.

\section{Proof of Theorem 2}

We will show that system (1) has no analytic first integrals. To do so, we need the following auxiliary result, it is due to Poincaré in [19], see also [6] for a direct proof. Through the paper $\mathbb{Z}^{+}$will denote the set of non-negative integers.

Theorem 7. Assume that the eigenvalues of the linear part at some singular point $p$ of the vector field $\chi, \lambda_{1}$ and $\lambda_{2}$ are non-zero and that they do not satisfy any resonance condition of the form

$$
\lambda_{1} k_{1}+\lambda_{2} k_{2}=0 \quad \text { for } \quad k_{1}, k_{2} \in \mathbb{Z}^{+} \quad \text { with } \quad k_{1}+k_{2}>0 .
$$

Then the vector field $\chi$ has no analytic first integrals defined in a neighborhood of $p$.

Proof of Theorem 2. We compute the singular points of system (1) and we get that these are $p=\left(a,\left(b+a^{2}\right) / a\right)$. Computing the eigenvalues $\lambda_{1}$ and $\lambda_{2}$ of the Jacobian matrix of system (1) at the fixed point $p$ we get that they are

$$
\lambda_{1,2}=\frac{1}{2}\left(b-1-a^{2} \pm \sqrt{-4 a^{2}+\left(1+a^{2}-b\right)^{2}}\right) .
$$

Suppose that there exists $k_{1}, k_{2} \in \mathbb{Z}^{+}$such that $k_{1} \lambda_{1}+k_{2} \lambda_{2}=0$. Note that by Theorem 7 if such integers do not exist we are done. Then $\lambda_{1}=-\alpha \lambda_{2}$ with $\alpha$ a positive rational, and hence in particular $\lambda_{1} \lambda_{2}=-\alpha \lambda_{2}^{2}<0$. But we have that $\lambda_{1} \lambda_{2}=a^{2}>0$. This completes the proof of Theorem 2.

\section{Proof of Proposition 3}

We first prove the part of the proposition concerning the existence of polynomial first integrals.

Lemma 8. System (2) has no polynomial first integrals.

Proof. We introduce the change of variables

$$
X=x, \quad Y=y+X
$$

and system (2) becomes

$$
X^{\prime}=-b-1+X Y-X^{2}, \quad Y^{\prime}=-1 .
$$

Since the change is linear, clearly it is equivalent to look for polynomial first integrals $h(x, y)$ of system (2) that to look for polynomial first integrals $\bar{h}(X, Y)=h(x, y)$ of system (7). We write $\bar{h}$ as a polynomial in the variable $X$, i.e.

$$
\bar{h}=\sum_{j=0}^{n} \bar{h}_{j}(Y) X^{j},
$$


where each $\bar{h}_{j}$ are polynomials in the variable $Y$. Since $\bar{h}$ is a polynomial first integral we can assume that $\bar{h}_{n} \neq 0$ and $n>0$, because if $n=0$ then $\bar{h}(X, Y)$ would be constant. Furthermore, it satisfies

$$
\left(-b-1+X Y-X^{2}\right) \frac{\partial \bar{h}}{\partial X}-\frac{\partial \bar{h}}{\partial Y}=0 .
$$

Computing in (9) the coefficient of $X^{n+1}$ we get

$$
-n \bar{h}_{n}(Y)=0 \text { that is } \bar{h}_{n}(Y)=0,
$$

a contradiction. This concludes the proof of the lemma.

To prove Proposition 3 we are left with the part of the Darboux polynomials with nonzero cofactor. To prove this part we will use the following result whose proof can be found in $[3,4]$.

Proposition 9. We suppose that $h \in \mathbb{C}[x, y]$ and let $h=h_{1}^{n_{1}} \cdots h_{r}^{n_{r}}$ be its factorization in irreducible factors over $\mathbb{C}[x, y]$. Then for a polynomial system (2) $h=0$ is an invariant algebraic curve with cofactor $K_{h}$ if and only if $h_{i}=0$ is an invariant algebraic curve for each $i=1, \ldots, r$ with cofactor $K_{f h i}$. Moreover $K_{h}=n_{1} K_{h_{1}}+\ldots+n_{r} K_{h_{r}}$.

Basically Proposition 9 states that to study the Darboux polynomials it is enough to consider the irreducible ones.

More precisely we will prove the following result.

Lemma 10. System (2) has an irreducible Darboux polynomial $h=h(x, y)$ with non-zero cofactor if and only if $b=0$, and in this case $h=y$ and the cofactor is $-x$.

Proof. Let $h=h(x, y)$ be an irreducible Darboux polynomial of system (2) with non-zero cofactor. We introduce the change of variables

$$
Y=y, \quad X=x+Y
$$

and write system (2) in the form

$$
X^{\prime}=-1, \quad Y^{\prime}=b-X Y+Y^{2},
$$

that can be written as

$$
\frac{d Y}{d X}=-b+X Y-Y^{2} .
$$

Then $\bar{h}=\bar{h}(X, Y)=h(x, y)$ is an irreducible Darboux polynomial of system (10) with non-zero cofactor $\bar{k}=\alpha_{0}+\alpha_{1} X+\alpha_{2} Y$ with $\alpha_{0}, \alpha_{1}, \alpha_{2} \in \mathbb{C}$ not all zero. Then $\bar{h}$ satisfies

$$
\frac{d \bar{h}}{d X}=\frac{\partial \bar{h}}{\partial X}+\frac{\partial \bar{h}}{\partial Y}\left(-b+Y X-Y^{2}\right)=\left(\alpha_{0}+\alpha_{1} X+\alpha_{2} Y\right) \bar{h}
$$

that is

$$
\frac{d \bar{h}}{d X}=\left(\alpha_{0}+\alpha_{1}(X-Y)+\left(\alpha_{1}+\alpha_{2}\right) Y\right) \bar{h}
$$

Now using that

we rewrite (11) as

$$
X-Y=\frac{1}{Y} \frac{d Y}{d X}+\frac{b}{Y}
$$

$$
\frac{d \bar{h}}{\bar{h}}=\alpha_{0} d X+\frac{\alpha_{1}}{Y} d Y+\left(\frac{b \alpha_{1}}{Y}+\left(\alpha_{1}+\alpha_{2}\right) Y\right) d X
$$

that is, after integrating and taking exponentials,

$$
\bar{h}=C Y^{\alpha_{1}} \exp \left(\alpha_{0} X+\left(\frac{b \alpha_{1}}{Y}+\left(\alpha_{1}+\alpha_{2}\right) Y\right) X\right),
$$


where $C$ is a constant. Since $\bar{h}$ must be a polynomial we have $\alpha_{0}=0, \alpha_{1}+\alpha_{2}=0$ and $b \alpha_{1}=0$. Then the cofactor of $\bar{h}$ is non-zero if and only if $b=0$, and in this case $\bar{h}$ is a polynomial of the form $\bar{h}=C Y^{\alpha_{1}}$. Since $\bar{h}$ is irreducible we get that $\alpha_{1}=1$. So we can take $\bar{h}=Y$ which yields $h=y$ and the cofactor $k=-x$. This completes the proof of the lemma.

Proof of Proposition 3. It follows directly from Lemmas 8 and 10.

\section{Proof of Proposition 4}

To prove Proposition 4 we will use the following known result whose proof and geometrical meaning is given in [5].

Proposition 11. The following statements hold.

(a) If $E=\exp (g / h)$ is an exponential factor for the polynomial system (2) and $h$ is not a constant polynomial, then $h=0$ is an invariant algebraic curve.

(b) Eventually $e^{g}$ for $k=1,2, \ldots$ can be exponential factors, coming from the multiplicity of the infinite invariant straight line.

Let $E=\exp (g / h)$ be an exponential factor of system (2). In view of Propositions 3 and 11, we have that $E=\exp (g)$ if $b \neq 0$, and $E=\exp \left(g / y^{n}\right)$ with $n \geq 0$ if $b=0$. When $n>0$ then $y \backslash$ Xg.

We first consider the case $E=\exp (g), g \in C[x, y]$. We have that the cofactor $L$ has degree one. We introduce the change of variables of (6), then $g(x, y)=\bar{g}(X, Y)$ and $\bar{g}$ satisfies

$$
\left(-b-1+X Y-X^{2}\right) \frac{\partial \bar{g}}{\partial X}-\frac{\partial \bar{g}}{\partial Y}=\beta_{0}+\beta_{1} X+\beta_{2} Y,
$$

for some $\beta_{0}, \beta_{1}, \beta_{2} \in \mathbb{C}$. We write $\bar{g}$ as a polynomial in the variable $X$ as in (8). Computing in (12) the coefficient of $X^{n+1}$ for $n \geq 1$ we get

$$
-n \bar{g}_{n}(Y)=0 \text { that is } \bar{g}_{n}(Y)=0 .
$$

Therefore $\bar{g}=\bar{g}_{0}(Y)$. Imposing that it satisfies (12) we get

$$
\frac{d \bar{g}_{0}}{d Y}=-\beta_{0}-\beta_{1} X-\beta_{2} Y .
$$

Solving it we get $\beta_{1}=0$ and $\bar{g}=-\beta_{0} Y-\beta_{2} Y^{2} / 2$. So we have the exponential factor $\exp (Y)$ with cofactor -1 and $\exp \left(Y^{2}\right)$ with cofator $-2 Y$.

Now we assume $b=0$ and that $E=\exp \left(\bar{g} /(Y-X)^{n}\right)$ with $n \geq 1$ and $\bar{g}$ satisfies

$$
\left(-1+X Y-X^{2}\right) \frac{\partial \bar{g}}{\partial X}-\frac{\partial \bar{g}}{\partial Y}=-n X \bar{g}+\left(\beta_{0}+\beta_{1} X+\beta_{2} Y\right)(Y-X)^{n},
$$

for some $\beta_{0}, \beta_{1}, \beta_{2} \in \mathbb{C}$. Denoting by $\hat{g}=\hat{g}(X)=\bar{g}(X, X)$ that is, evaluating it on $Y=X$ we get that it satisfies

$$
\frac{d \hat{g}}{d X}=n X \hat{g}
$$

So $\hat{g}=C e^{n X^{2} / 2}$ with $C$ a constant. Since $\hat{g}$ is a polynomial it follows that $C=0$. So $\hat{g}=0$, and $Y-X$ divides $\bar{g}(X, Y)$. Consequently $y \mid g(x, y)$ a contradiction. This completes the proof of the proposition. 


\section{Proof of Theorem 5}

We first recall that a non-constant complex function $R: \mathbb{C}^{2} \rightarrow \mathbb{C}$ is an integrating factor of the polynomial vector field $\chi$ on $U$, if one of the following three equivalent conditions holds

$$
\frac{\partial(R P)}{\partial x}=-\frac{\partial(R Q)}{\partial y}, \quad \operatorname{div}(R P, R Q)=0, \quad \chi R=-R \operatorname{div}(P, Q),
$$

on $U$ with $P=-b-1+x y$ and $Q=b-x y$. As usual the divergence of the vector field $\chi$ is given by

$$
\operatorname{div}(P, Q)=\frac{\partial P}{\partial x}+\frac{\partial Q}{\partial y}=y-x .
$$

From the Darboux theory of integrability we have the next result proved, for instance, in [4].

Theorem 12. Suppose that the polynomial vector field $\chi$ of degree $m$ defined in $\mathbb{C}^{2}$ admits $p$ invariant algebraic curves $f_{i}=0$ with cofactors $K_{i}$ for $i=1, \ldots, p$, and $q$ exponential factors $E_{j}=\exp \left(g_{j} / h_{j}\right)$ with $\left(g_{j}, h_{j}\right)=1$ and cofactors $L_{j}$ for $j=1, \ldots, q$. Then there exist $\lambda_{i}, \mu_{j} \in \mathbb{C}$ not all zero such that

$$
\sum_{i=1}^{p} \lambda_{i} K_{i}+\sum_{j=1}^{q} \mu_{j} L_{j}=-\operatorname{div}(P, Q)
$$

if and only if the function of Darboux type

$$
f_{1}^{\lambda_{1}} \cdots f_{p}^{\lambda_{p}} E_{1}^{\mu_{1}} \cdots E_{q}^{\mu_{q}}
$$

is an integrating factor of the vector field $\chi$.

To prove the results related with Liouvillian first integrals we use the following result proved in $[2,20]$.

Theorem 13. The polynomial differential system (2) has a Liouvillian first integral if and only if it has an integrating factor of Darboux type (see (13)).

By Proposition 3 system (2) has a Darboux polynomial if and only if $b=0$ (in which case it is $y$ with cofactor $K_{1}=-x$ ), and by Proposition 4 it has the exponential factors $\exp (x+y)$ and $\exp \left((x+y)^{2}\right)$ with cofactors $L_{1}=-1$ and $L_{2}=-2(x+y)$, respectively.

In order that system (2) has a Liouvillian first integral, by Theorem 13, system (2) must have an integrating factor of Darboux type. From Theorem 12 system (2) has an integrating factor of Darboux type if and only if

$$
\lambda_{1} K_{1}+\mu_{1} L_{1}+\mu_{2} L_{2}=-\lambda_{1} x-\mu_{1}-2 \mu_{2}(x+y)=-(y-x), \quad \lambda_{1}, \mu_{1}, \mu_{2} \in \mathbb{C} .
$$

This equality is possible if and only if $\mu_{1}=0, \lambda_{1}=-2$ and $\mu_{2}=-1 / 2$. Therefore, the integrating factor is $y^{-2} e^{(x+y)^{2} / 2}$. Computing the first integral associated to this integrating factor we get the one stated in Theorem 5 . This completes the proof of the theorem.

\section{ACKNOWLEDGEMENTS}

The first author is supported by the grants MICINN/FEDER MTM 2008-03437, AGAUR 2009SGR410 and by ICREA Academia. The third author is partially supported by FCT through CAMGDS, Lisbon. 


\section{REFERENCES}

[1] M. Abramowitz And I.A. Stegun, Handbook of mathematical functions with formulas, graphs, and mathematical tables, National Bureau of Standards Applied Mathematics Series, 55, U.S. Government Printing Office, Washington, D.C., 1964.

[2] C. Christopher, Liouvillian first integral of second order polynomial differential systems, Electronic J. of Differential Equations 1999 (1999), 1-7.

[3] L. Cairó, H. Giacomini And J. LliBre, Liouvillian first integrals for the planar Lotka-Volterra system, Rend. Circ. Mat. Palermo 52 (2003), 389-418.

[4] C. Christopher And J. Llibre, Integrability via invariant algebraic curves for planar polynomial differential systems, Ann. Diff. Equations 16 (2000), 5-19.

[5] C. Christopher, J. Llibre and J.V. Pereira, Multiplicity of invariant algebraic curves and Darboux integrability, Pacific J. of Math. 229 (2007), 63-117.

[6] S. D. FURTA, On non-integrability of general systems of differential equations, Z. Angew. Math. Phys. 47 (1996), 112-131.

[7] Yu. A. Kuznetsov, SpringerElements of Applied Bifurcation Theory, Second Edition, Appl. Math. Sci. 112, Springer-Verlag, 2000.

[8] S. Labrunie, On the polynomial first integrals of the $(a, b, c)$ Lotka-Volterra system, J. Math. Phys. 37 (1996), 5539-5550.

[9] J. Llibre AND C. VAlls, Global analytic first integrals for the real planar Lotka-Volterra system, J. Math. Phys. 48 (2007), no. 3, 033507, 13 pp.

[10] J. LliBRE AND C. VALls, Global analytic first integrals for the simplified multistrain/two-stream model for tuberculosis and dengue fever, J. Nonlinear Math. Phys. 16 (2009), 505-516.

[11] J. Llibre And C. VAlls, Liouvillian first integrals for Liénard polynomial differential systems, Proc. Amer. Math. Soc. 138 (2010), no. 9, 3229-3239.

[12] J. Llibre And C. VAlls, Liouvillian integrability of the FitzHugh-Nagumo systems, J. Geom. Phys. 60 (2010), no. 12, 1974-1983.

[13] J. LliBre AND C. VALls, On the local analytic integrability at the singular point of a class of Liénard analytic differential systems, Proc. Amer. Math. Soc. 138 (2010), no. 1, 253-261.

[14] J. LliBRE AND C. VALLS, Liouvillian first integrals of quadratic-linear polynomial differential systems, J. Math. Anal. Appl. 379 (2011), no. 1, 188-199.

[15] J. Moulin Ollagnier, Polynomial first integrals of the Lotka-Volterra system, Bull. Sci. math. 121 (1997), 463-476

[16] J. Moulin Ollagnier, Rational integration of the Lotka-Volterra system, Bull. Sci. math. 123 (1999), $437-466$.

[17] J. Moulin Ollagnier, Liouvillian Integration of the Lotka-Volterra system, Qual. Theory Dyn. Syst. 2 (2001), 307-358.

[18] J. Moulin Ollagnier, Corrections and complements to: "Liouvillian integration of the Lotka-Volterra system" [Qual. Theory Dyn. Syst. 2 (2001), 307-358, Qual. Theory Dyn. Syst. 5 (2004), $275-284$.

[19] H. Poincaré, Mémoire sur les courbes définies par les équations différentielles (Oeuvreus de Henri Poincaré, I). Gauthiers-Villars, Paris, 1951, pp. 95-114.

[20] M. F. Singer, Liouvillian first integrals of differential systems, Trans. Amer. Math. Soc. 333 (1992), $673-688$.

1 Departament de Matemàtiques, Universitat Autònoma de Barcelona, 08193 Bellaterra, Barcelona, Catalonia, Spain

E-mail address: jllibre@mat.uab.cat

2 Departamento de Matemática, Instituto Superior Técnico, Universidade Técnica de Lisboa, Av. Rovisco Pais 1049-001, Lisboa, Portugal

E-mail address: cvalls@math.ist.utl.pt 